\section{Customer Preferences and Opaque Intermediaries}

Cornell Hospitality Quarterly $1-12$

(C) The Author(s) 2017 Reprints and permissions: sagepub.com/journalsPermissions.nav DOI: $10.1177 / 1938965516686108$ journals.sagepub.com/home/cqx (S)AGE

\author{
Xiaoqing (Kristine) Xie', Chris K. Anderson ${ }^{2}$, and Rohit Verma ${ }^{2}$
}

\begin{abstract}
Using two choice-based experiments, we evaluate consumer preferences hotel attributes for firms selling hotel rooms across three online distribution channel formats: full information, semi-opaque, and opaque online travel agents. A multinomial logit model is used to analyze the experimental data and measure consumer trade-offs between price and other product attributes. We then use these preferences to determine optimal channel selling strategies. Our optimal channel strategies illustrate under what conditions firms should add opaque distribution channels and the resulting incremental revenue obtained with the setting of optimal channel specific prices. We deploy two choice-based experiments, traditional and menu-based, in an effort to add flexibility to survey respondents in choice selection. As part of our analysis, we compare managerial insights from analysis based on traditional choice-based experiments to that using menu-based choice experiments. In general, we indicate that both forms of opaque selling increase firm demand and that with appropriate pricing can also increase firm revenue. In addition, opaque channels have elevated price sensitivity and increased impact of guest reviews versus traditional online travel agents.
\end{abstract}

\title{
Keywords
}

online choice experiment, opaque selling, the MNL model, buyer behavior

\section{Introduction}

The selling of services (rooms, rental cars, airline seats, etc.) online has dramatically changed how service firms reach customers, with online travel sales now exceeding offline (or traditional sales channels). Although adding online selling channels should provide firms an opportunity to expand the market and achieve a finer level consumer segmentation, there exists very little empirical research evaluating the impacts of additional online sales channels upon firm revenue. To this end, we use an online choice experiment to assess the revenue impacts of a firm adopting a multichannel selling strategy whereby the channels differ in the level of product information provided to the consumer. We use online hotel booking as an illustration as hotels have deployed online channel strategies for almost 20 years. The online distribution channels we investigate include a regular full information online travel agent (OTA), such as Expedia, Orbitz, or Travelocity; a fully opaque channel like Priceline's name-your-own-price (NYOP) model; and less opaque channels which we term semi-opaque or opaque posted price channel like Hotwire or Lastminute. Semi-opaque and opaque channels, unlike regular full information OTAs, offer customers products or services with aspects of the service provider concealed until the transaction has been completed requiring consumers to make price-information trade-offs. Opaque selling is common across all facets of travel; with air travel, the consumer is unaware of the itinerary (connections and layover durations) or airline and with rental cars, the consumer does not know the type of car or rental firm until after paying for the service. The level of opacity or uncertainty varies across the different opaque channels as some choose to offer cancelation opportunities as in the case of Lastminute.com, provide user generated feedback (review scores), or list some of the hotel amenities as in the case of Hotwire.com. Similarly the degree of opacity may also be impacted by the market, as markets with fewer similar competitors offer decreased opacity over markets with a large number of similar service providers. Opaque selling has been deployed as a business practice for almost 20 years with the launch of Priceline's NYOP in 1997 followed shortly by Hotwire's posted price format in 2000, but research on opaque selling is relatively new. In the following, we highlight aspects of opaque selling but focus largely on the opaque selling literature which we build upon. In addition to the opaque selling literature, we briefly mention

\footnotetext{
'China Europe International Business School (CEIBS), China
}

${ }^{2}$ Cornell University, Ithaca, NY, USA

\section{Corresponding Author:}

Xiaoqing (Kristine) Xie, Department of Decision Sciences, China Europe International Business School (CEIBS), Shanghai, China.

Email: xkristine@ceibs.edu 
the experimental choice literature relevant to our methodological contributions.

\section{Simultaneously Selling on Both Opaque and Regular Channels}

There are two main forms of opaque selling - opaque selling with bidding pioneered by Priceline's NYOP model and semi-opaque selling or opaque selling with posted prices. Anderson (2009) provides a detailed description of Priceline's NYOP model and illustrates methods for determining optimal bid policies on Priceline. Whereas Anderson and Xie (2012) provide background into Hotwire's semiopaque posted price model as part of their development of optimal prices for firms deploying Hotwire as a selling format.

Early opaque selling research has been analytical in nature with attempts to theoretically justify the use of opaque selling (alongside full information selling). Fay (2008) uses a traditional hotelling model to study a game between two service providers selling products to two types of customers (loyals and searchers) on both an opaque posted price channel and a traditional distribution channel. Fay shows that opaque selling benefits the monopoly service provider when customers have heterogeneous values for products. Shapiro and Shi (2008) extend the model of Fay (2008) to $N$ firms with the number of firms indicating the degree of opacity-uncertainty in knowledge of service provider increases with number of firms. Shapiro and Shi focus on providing a rational for opaque selling. In essence, these papers illustrate (analytically) why service providers are willing to distribute products through opaque travel sites such as Priceline and Hotwire.

Similar to our article, Shapiro and Zillante (2009) implement an experimental approach to exam the impacts of opaque and full information selling. While we look at posted opaque prices in concert with opaque bidding (in addition to full information), Shapiro and Zillante focus only on opaque bidding and full information selling. Shapiro and Zillante also aim to study the properties of NYOP mechanism and especially whether NYOP websites such as Priceline should use the opaque feature. Similar to our results, they show adding a NYOP selling mechanism with the opaque feature to the regular selling channel will decrease the seller profit when the bidding threshold (i.e., the "price" on bidding channel) is set too low. Granados, Gupta, and Kauffman (2008) use sales data from an OTA to estimate elasticity differences between transparent and opaque selling of airline tickets. Using aggregated transactional data, they indicate the opaque products are more elastic than transparent tickets. As they use aggregate level data, they exclude numerous critical attributes of airline tickets including carriers, trip duration, stops, and so on, all features which one would consider critical in differentiating opaque versus transparent (or full information) selling. Similar to Shapiro and Zillante (2009), Granados et al. (2008) do not differentiate opaque posted price selling from opaque bidding. In a follow-on paper, Granados, Gupta, and Kauffman (2012) add additional transactional data from traditional (offline) travel agents to their sample, they find that sales are less elastic at traditional agents, in fact sales at traditional travel agents they find to be more inelastic than sales at transparent OTAs and opaque OTAs more elastic than transparent OTAs. Similar to their 2008 paper, the results are for aggregated sales data and may not be reflective of true demand as the data excludes nonpurchase events, product attributes and sales through other channels (airline direct, wholesale, etc.).

So while research on opaque selling has over a 10 -year history, very few papers actually fully investigate all aspects of opaque and full information selling, in fact Anderson and Xie (2014) is the only paper that investigates a firm using two opaque (posted prices and bidding) channels simultaneously with regular full information posted price channels. Anderson and Xie (2014) develop endogenous consumer segments where consumers choose the channel of their choice by maximizing their surplus (utility) using a stylized model of consumer behavior. Then, based on this exogenous channel choices, Anderson and Xie develop optimal channel prices and illustrate revenue impacts from opaque selling owing to consumer segmentation.

We extend this earlier research in numerous ways; first, we empirically assess the analytical insights of Anderson and Xie (2014) as well as extend the results of Shapiro and Zillante (2009) and those of Granados et al. $(2008,2012)$ by differentiating opaque posted prices from opaque bidding in addition to selling via transparent or full information selling. In addition, most of this prior research has been set in the airline industry. The airline industry tends to be much more commoditized (less differentiated than hotels) and as such we would expect the impacts of opaque selling to be different from that of a highly differentiated industry like hotels. In addition, our methodology, discrete choice experimental analysis, as opposed to the aggregated market response model used in Granados et al. $(2008,2012)$ allows estimation of the impacts of product attributes as well as true demand given we have a no-purchase option. In the following section, we provide some further background into our experimental methods.

\section{Experimental Choice Analysis}

In the past four decades, conjoint analysis has become the most widely applied method for measuring and analyzing consumer behavior and preferences. Experimental choice analysis, is a natural extension to conjoint analysis. Louviere (1992) offers a review on the state of art in experimental choice analysis. Traditional experimental choice analysis 
allows users to select among a set of alternatives each with potentially different levels across a set of attributes. Traditional choice analysis fails to capture the flexibility in today's world of mass customization where consumers are often allowed to customize their purchase by both attributes and levels. Liechty, Ramaswamy, and Cohen (2001) propose subtle changes to the traditional choice experiment where respondents customize their choices versus simply select from a series of options. Like a traditional choice experiment, respondents select among levels of attributes across features but in addition there exists a set of feature menus that users select from. Attractiveness or sensitivity of features is estimated as respondents see differing menus of features with different available levels of the attributes. More recent menu-based approaches to choice experiments have dealt with solving some of the methodological issues associated with menu-based designed as measurement needs to either assume that attributes are independent of the features available or suffer from the curse of dimensionality if all the potential interactions created by partial availability of features during the experiment (see Kamakura \& Kwak, 2010 , for a recent review and discussion of menu-choice modeling). Here, we contribute to the experimental choice literature by utilizing two experimental approaches, traditional choice experiments and menu-based. We compare parameter estimates under both frameworks but more importantly we compare firm decisions and revenue impacts that results under use of models estimated under the two choice settings.

To evaluate the revenue impacts of a multichannel strategy, we deploy online choice-based experiments. We use two survey formats: a traditional experimental choice survey, and a second a menu variant to add flexibility to the consumer choice process allowing us to contrast managerial insights under the two survey formats. A multinomial logit (MNL) model is used to assess the impacts of channel and hotel attributes (such as price, star rating, previous guest rating, etc.) upon purchase behavior. We then use hotel and channel attributes to illustrate optimal channel prices and the resulting incremental revenues. Our article makes contributions on two fronts: To our knowledge, ours is the only article that empirically quantifies consumer trade-offs across the three major forms of online selling in hospitality and then uses this to determine the incremental revenue created by multichannel use. Hotels can then use our insights to decide whether they should adopt an opaque selling strategy or whether the current channel prices (in combination with their attributes) indicate that opaque selling would not result in incremental revenue. As part of this quantification, we provide additional insight into how attributes such as star level, price, and review scores differ in their impact as a function of the opacity of the sales channel providing further direction for how firms might capitalize on opaque selling formats.
Secondarily, we contribute to the experimental discrete choice analysis literature as we contrast insights generated from the use of traditional choice-based experiments to those of menu-based choice experiments indicating that small changes in survey format can result in substantive changes in managerial insight.

\section{Data Collection and Experimental Design}

Respondents were presented with three hotel-channel options along with a no-purchase option. Each respondent, in addition to the no-purchase option, sees three listings: a regular full information listing similar to what one would see at an OTA or suppliers website, a posted price opaque travel site, and an opaque bidding travel site (subsequently referred to as REG, OPQ, and BID, respectively). Prior to seeing the choice scenarios, respondents are asked to read educational information about the three forms of online selling.

\section{Alternative Attributes}

Respondents were shown 10 choice scenarios (each with the four options). A sample set of choice scenarios is shown in Figure 1 with attributes and levels summarized in Table 1. To clearly communicate the differences in hotel-channel options on the online questionnaire, we label the options "Full Information," "Partial Information," and "Partial Information With Bidding" (representing REG, OPQ, and BID, respectively).

During the early stages, respondents are asked to select from a list any hotel loyalty programs to which they belong so that during the experiment, they are shown hotels (in the full information listing) both within and outside those programs in an effort to evaluate the impact of loyalty programs upon purchase and channel choice. The REG hotel listing has three additional attributes. The star rating of the hotel is varied among three levels $3,31 \frac{1}{2}$, and 4 stars. Hotel prices for the REG are based on average prices listed at Expedia across hotel brands, star levels, and locations. Then during the survey, REG prices were at levels of 0.86 , $0.93,1.15$, and 1.3 times average prices. The last attribute of a REG hotel listing is the previous guest rating which has four levels: $3.5,4,4.5$, and 4.8 (on 5-point scale).

Four attributes are used to describe an OPQ hotel listing. The first attribute is the star rating of the OPQ property which has four levels $3,3 \frac{1}{2}, 4$, and $4 \frac{1}{2}$ stars. The second attribute of an OPQ hotel listing is the price discount $(15 \%$, $25 \%, 35 \%$, and $45 \%$ ) off the average price of the corresponding (i.e., same star level) REG hotel listing. Last, OPQ listing may or may not display previous guest review scores (attribute three), and if shown, the score can take one of four levels: 3.5, 4, 4.5, and 4.8 on a 5-point scale. 


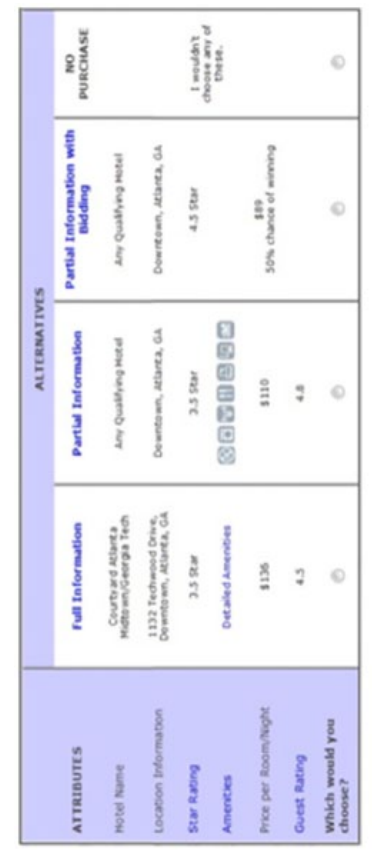

(a)

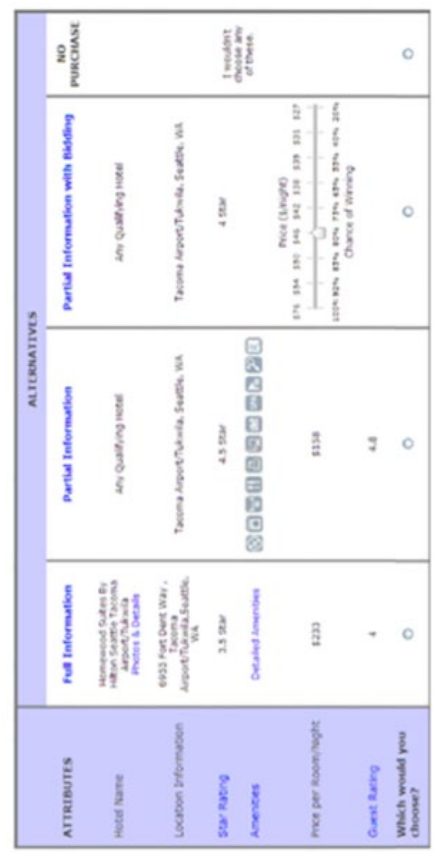

(b)
Figure 1.

Experimental Screen Layout: (a) Traditional Choice Scenario and (b) Menu Choice Scenario.

Table 1.

Attributes and Their Levels.

\begin{tabular}{|c|c|c|}
\hline Alternative & Attribute & Levels \\
\hline \multirow{3}{*}{$\begin{array}{l}\text { Full } \\
\text { Information }\end{array}$} & Star rating & $3,31 / 2,4$ \\
\hline & Price multiplier & $0.86,0.93,1.15,1.3$ \\
\hline & Guest rating & $3.5,4,4.5,4.8$ \\
\hline \multirow{4}{*}{$\begin{array}{l}\text { Partial } \\
\text { Information }\end{array}$} & Star rating & $3,31 / 2,4,41 / 2$ \\
\hline & Price discount & $45 \%, 35 \%, 25 \%, 15 \%$ \\
\hline & Guest rating & shown, not shown \\
\hline & Guest rating if shown & $3.5,4,4.5,4.8$ \\
\hline \multirow{6}{*}{$\begin{array}{l}\text { Partial } \\
\text { Information } \\
\text { With } \\
\text { Bidding }\end{array}$} & Star rating & $3,31 / 2,4,41 / 2$ \\
\hline & Traditional & \\
\hline & Price discount & $65 \%, 55 \%, 45 \%$ \\
\hline & Chance of winning & $95 \%, 50 \%$ \\
\hline & Menu-based & \\
\hline & $\begin{array}{l}\text { Price discount } \\
\text { (Chance of winning) }\end{array}$ & $\begin{array}{c}80 \%(100 \%), 70 \%(92 \%), \\
65 \%(85 \%), 60 \%(80 \%), \\
55 \%(75 \%), 50 \%(65 \%), \\
45 \%(55 \%), 40 \%(40 \%), \\
35 \%(20 \%)\end{array}$ \\
\hline
\end{tabular}

The BID alternative is characterized by star level and price (or bid). The star levels of the BID option are from the same levels as the OPQ option $\left(3,3 \frac{1}{2}, 4\right.$, or $4 \frac{1}{2}$ stars) but a choice scenario may display differing star levels across OPQ and BID (and REG), for example, a 3 star OPQ listing alongside a 4 star BID option. The second attribute of a BID hotel listing is the bidding price discount. To do a choicebased experimental analysis, we do not allow respondents to enter their own bid offer on the screen. Instead, we show a price along with a winning probability to imitate the bidding process. We facilitate this bid process in two manners: a traditional choice-based experiment with fixed levels and a menu-based choice experiment where users select their bids freely via a slider. We further elaborate on the bid prices and probabilities for the two experimental options in the following sections.

\section{Traditional Experimental Choice}

The BID option prices are at discounts of $45 \%, 55 \%$, or $65 \%$ of the corresponding average REG prices. BID discounts are lower than OPQ listing as consumers not only deal with product uncertainty (similar to OPQ listings) they also have uncertainty around acquisition of capacity (will their bid be successful). BID prices also have an associated chance of winning (the third attribute), which is the possibility of getting the hotel room with the bid. Winning probabilities are set at either $95 \%$ or $50 \%$ chance of winning.

\section{Menu Experimental Choice}

Figure $1 \mathrm{~b}$ shows a sample survey screen for the menu-based variant. The layout is the same as shown in Figure 1a except for the addition of the slider under the BID option. Under this form of the survey, respondents can move the slider to specify the bid/probability of winning combination at which they are interested purchasing via the opaque bidding format.

Our bid/probability experimental formats are based on input screens used by Priceline. Our traditional choice analysis format is based on Priceline's bid guidance provided for rental cars as shown in Figure 2a. As illustrated in the figure, Priceline provides two recommended bids: one with a good chance (our 50\%) and the other a great (our 95\%) chance of being accepted. Whereas Figure $2 b$ shows Priceline's hotel bid price guidance slider upon which we base our menu bid/probability format.

We purchased a sample of 1,045 respondents through a survey sampling company. A total of 531 respondents completed the traditional format survey with 514 completing the menu-based choice experiment. Each respondent was shown 10 choice scenarios, and so we collected 10,450 experimental observations. Table 2 summarizes the choice percentages for each of the alternatives for both survey formats. Both survey formats show similar percentages with opaque channels being most attractive to consumers and consumers preferring the bidding option (BID) over posted opaque prices (OPQ) when given the flexibility in the menu-based survey. The choice experiment data were 


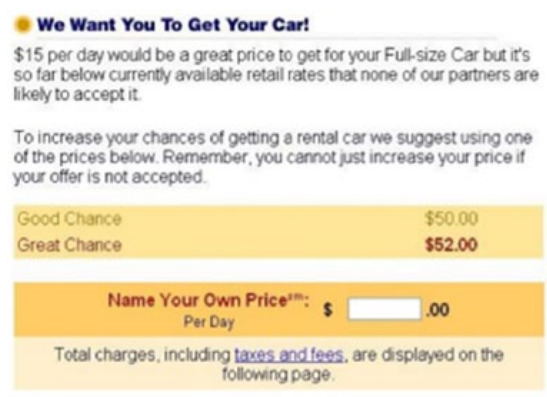

(a)

\section{Enter Your Bid}

You're bidding on a 3-Star hotel in the Downtown Ithaca area

\section{$\$ 128$}

The median retail price for a similar hotel is $\$ 161$

(b)

\section{Figure 2.}

Priceline Bidding Insight: (a) Rental Car Bidding and (b) Priceline App Bidding.

Table 2.

Market Share (\%) by Alternative.

\begin{tabular}{lcccc}
\hline Alternative & REG & OPQ & BID & No Purchase \\
\hline Traditional & 15.16 & 25.57 & 30.72 & 28.55 \\
Menu & 14.40 & 26.81 & 33.48 & 25.31 \\
\hline
\end{tabular}

analyzed using maximum likelihood estimation via a MNL model as discussed in detail in the next section.

\section{Model Specification, Estimation, and Analysis Results}

In a discrete choice model, an individual decision maker (consumer) makes a choice among a feasible set of alternatives. The decision is often made by maximizing random utilities of the alternatives. The MNL model is the most commonly used random utility model. For a greater detail in discrete choice modeling or the MNL model, please refer to Ben-Akiva and Lerman (1985). We provide a brief background into the MNL model to fully understand our novel approach to the estimation/calibration of the no-purchase utility which serves a critical role in how we estimate the incremental demand and revenue from opaque selling.

In an MNL model, we usually consider a universal set of alternatives denoted by $C$. The set of alternatives for consumer $n$ is denoted by $C_{n} \in C$. $U_{n i}$ denotes the random utility of alternative $i$ for consumer $n$, which can be divided into two addictive components: $U_{n i}=V_{n i}+\varepsilon_{n i}$, where, $V_{n i}$ is the deterministic (representative) component. $\varepsilon_{m i}$ is the random component and $\varepsilon_{n i}$ s are independent and identically Gumbel distributed.

Each alternative $i$ in a choice set $C_{n}$ is often characterized by a finite set of observable attributes $x_{n i 1}, x_{n i 2}, \ldots, x_{n i k}$ in the following way:

$$
V_{n i}=\beta^{t} \mathbf{x}_{n i},
$$

where $\mathbf{x}_{n i}=\left(x_{n i 1}, x_{n i 2}, \ldots, x_{n i k}\right)^{t}$ and $\boldsymbol{\beta}_{i}=\left(\beta_{i 1}, \beta_{i 2}, \ldots, \beta_{i k}\right)^{t}$ are the vectors of unknown parameters that need to be estimated.

Every consumer is a utility maximizer, so the choice probability of alternative $i \in C_{n}$ is given by

$$
\begin{aligned}
P_{n}(i) & =\operatorname{Pr}\left[U_{n i} \geq U_{n j}, \text { all } j \in C_{n} \text { for } \forall n\right] \\
& =\frac{e^{V_{n i}}}{\sum_{j \in C_{n}} e^{V_{n j}}} \\
& =\frac{e^{\beta_{i}^{\prime} \mathbf{X}_{n i}}}{\sum_{j \in C_{n}} e^{\beta^{\prime} \mathbf{X}_{n j}}} .
\end{aligned}
$$

We estimate the parameters $\beta_{i}$ and obtain the choice probabilities by applying maximum likelihood method.

The corresponding likelihood function is:

$$
L=\prod_{n=1}^{M} \prod_{i \in C_{n}}\left[P_{n}(i)\right]^{y_{n i}},
$$

where $y_{n i}=1$, if consumer $n$ chooses alternative $i \in C_{n}$, 0 otherwise; $M=$ the total number of observations (transactions).

In our MNL, the respondent makes a choice among the four alternatives in the choice set $C=\{$ REG, OPQ, BID, No Purchase $\}$. The decision is made by maximizing random utilities of the alternatives.

The utility associated with the first three alternatives for respondent $n$ is given by

$$
U_{n i}=\boldsymbol{\beta}_{i}^{\prime} \mathbf{x}_{n i}+\varepsilon_{n i},
$$

where $i=1,2,3$ represents the alternatives REG, OPQ, and BID, respectively; $\mathbf{x}_{n i}$ are the vectors including attributes for alternative $i$ and the characteristics of consumer $n ; \boldsymbol{\beta}$ are the corresponding vectors of parameters to be estimated using the MNL model. 
By Equation 2, the probability $P_{n}(i)$ respondent $n$ chooses alternative $i$ is given as the follows:

$$
P_{n}(i)=\frac{e^{\beta^{\prime} \mathbf{X} n i}}{\sum_{j=1}^{j=3} e^{\beta_{j}^{\prime} \mathbf{X}_{n j}}+e^{U_{00}}},
$$

where $i=1,2,3$ represents the alternatives REG, OPQ, and BID, respectively. $U_{00}$ represents the utility of "No Purchase" choice. The "No Purchase" choice probability is

$$
P_{00}=\frac{e^{U_{00}}}{\sum_{j=1}^{j=3} e^{\beta_{j}^{\prime} \mathbf{X}_{n j}}+e^{U_{00}} .}
$$

Note that using an MNL model requires alternatives in the choice set to have the independence of Irrelevant Alternatives (IIA) property which holds that changes in the choice set do not affect the ratio of choice probabilities of any two alternatives. As one can see that in Equation 2, $\sum_{i \in C n} P(i)=1$, the IIA property implies if some alternatives are removed or added from the choice set $C$, the choice probabilities $P_{n}(i)$ will be proportionally revised so that they can still be summed up to be 1 (Ratliff, Rao, Narayan, \& Yellepeddi, 2008). Specifically, in our situation, when we add either the OPQ hotel listing or the BID hotel listing (or both) to the REG hotel listing, we can compute the proportionally shifted new choice probabilities of each alternative, allowing estimation of expected incremental revenue.

As one can see in Equations 5 and 6, we face the issue of properly estimating the utility of the "No Purchase" choice $\left(U_{00}\right)$. We assume that there are enough hotels in the market such that "No Purchase" consumers actually purchase either from other hotels (competitors) or this service provider's offline distribution channels such as walk-ins and call centers. Under this assumption, analogous to Ratliff et al. (2008) which provides a recapture heuristic for estimating unconstrained demand from airline bookings, we estimate the no-purchase utility by using the actual market share of the three online distribution channel reported by STR (Smith Travel Research) Global in 2010. In the report, OTA market share is $4.6 \%$, opaque OTA market share is $2 \%$, and the hotel website has $17.5 \%$ market share. Assume that we know or can estimate our own market share from the historical sales data and denote it by $d$. We can approximate the hotel's demand on REG channel to be the sum of the OTA demand and the hotel's website demand multiplied by $d$, as these two distribution channels both reveal full information including identity of the hotel. Opaque OTA demand is the estimate for demand of the two opaque listings. Hence, the three hotel listings' market share is approximately $M S_{h}=$ $(4.6 \%+17.5 \%) d+2 \%=22.1 \% d+2 \%$. Then the competitors' market share plus our own hotel's offline market share is $M S=1-M S_{h}$, and this is the simplified estimate of the likelihood of the "No Purchase" choice $P_{00}$. Therefore, by substituting it into Equations 5 and 6, we get a simple estimate for "No Purchase" utility $U_{00}$ which can be solved from the equation below

$$
\begin{aligned}
e^{U_{00}} & =\frac{M S_{c}}{M S_{h}} \sum_{j=1}^{j=3} e^{\beta_{j}^{\prime} \mathbf{X}_{n j}} \\
& =\frac{1-M S_{h}}{M S_{h}} \sum_{j=1}^{j=3} e^{\beta_{j}^{\prime} \mathbf{X}_{n j} .}
\end{aligned}
$$

Plugging Equation 7 and $M S_{c}=1-M S_{h}$ back into Equation 5, we have the choice probabilities for all three alternatives $i=1,2,3$ (REG, OPQ, BID, respectively) are given by the following:

$$
P_{n}(i)=M S_{h} \frac{e^{\beta_{i}^{\prime} \mathbf{X}_{n i}}}{\sum_{j=1}^{j=3} e^{\beta_{i j}^{\prime} \mathbf{X}_{n j}}} .
$$

\section{Model Estimation Results and Analysis}

Table 3 presents parameter estimates for both survey formats. In the following sections, we focus our discussion around using parameter estimates from the traditional choice-based results and later discuss the differences in parameters and the resulting impacts of traditional versus menu-based formats. As a simple check of model fit, we compare the $\log$ likelihood $L L$ of the estimated model (-6761.517) versus that of a simplified model LL* with channel (BID, OPQ, REG) specific constants (-7995.938). To determine whether the estimated model is superior to its reference model, the $-2\left(L L-L^{*}\right)$ value obtained is compared with a chi-square statistic with degrees of freedom (20 in our model) equal to the difference in the number of parameters estimated for the two models (Hensher et al., 2005) producing a $p$ value of .000 , as a result we reject the null hypothesis that the estimated model is no better than the reference model.

In the first section of the questionnaire, we asked which (if any) loyalty program(s) the respondent was a member of, and then conditioned the choice sets to include some options with their brand of choice, allowing estimation of Loyal to REG hotel during model fitting. This is a dummy variable coded as 1 if the REG alternative is from a hotel chain whose loyalty program that the respondent is affiliated to, 0 otherwise. Its positive coefficient estimate implies consumers who are in loyalty programs are more likely to choose the hotels from that brand (vs. other brands or independents).

The price variable for each channel is coded as a standardized continuous variable, that is, the difference between the actual price that the respondent saw and the average price across all price values shown on that channel divided by the standard deviation of all prices. Price coefficients are 
Table 3.

MNL Parameter Estimation Results.

\begin{tabular}{|c|c|c|c|c|}
\hline \multirow[b]{2}{*}{ Variable Name } & \multicolumn{2}{|c|}{ Traditional } & \multicolumn{2}{|c|}{ Menu } \\
\hline & Parameter Estimate & SE & Parameter Estimate & SE \\
\hline Loyal to the REG hotel (REG) & $0.8639 * * *$ & 0.17 & $0.4129 *$ & 0.1899 \\
\hline REG constant & $-1.2362 * * *$ & 0.3495 & $-0.9879 * *$ & 0.3748 \\
\hline OPQ constant & $-0.454 * * *$ & 0.1201 & $-0.5077^{* * * *}$ & 0.1031 \\
\hline BID constant & 0.0935 & 0.0887 & $1.1005^{* * *}$ & 0.0948 \\
\hline REG price & $-0.4034 * * *$ & 0.0888 & $-0.4253^{* * * *}$ & 0.0913 \\
\hline OPQ price & $-0.6055^{* * *}$ & 0.0868 & $-0.3677^{* * * *}$ & 0.0406 \\
\hline BID price & $-0.6799 * * *$ & 0.0758 & $-2.1595^{* * * *}$ & 0.1273 \\
\hline REG 3 star & 0.0875 & 0.141 & -0.0947 & 0.1493 \\
\hline REG 3.5 star & 0.0796 & 0.121 & 0.1017 & 0.13 \\
\hline OPQ 3 star & 0.0475 & 0.158 & $0.1909 *$ & 0.0796 \\
\hline OPQ 3.5 star & 0.0863 & 0.1275 & -0.0034 & 0.1528 \\
\hline OPQ 4 star & 0.1058 & 0.1178 & $0.2869 *$ & 0.1169 \\
\hline BID 3 star & -0.0258 & 0.1522 & $-4.0342 * * *$ & 0.2536 \\
\hline BID 3.5 star & 0.0339 & 0.1111 & $-2.124 \mid * * *$ & 0.167 \\
\hline BID 4 star & 0.044 & 0.106 & $-0.8419 * * *$ & 0.1244 \\
\hline REG 3 star $\times$ price & $-0.4293 * *$ & 0.1449 & $-0.5468 * * *$ & 0.1504 \\
\hline REG 3.5 star $\times$ price & $-0.258 I^{*}$ & 0.1299 & -0.2198 & 0.1274 \\
\hline OPQ 3 star $\times$ price & $-0.3577^{*}$ & 0.1606 & $-0.9828 * * *$ & 0.1581 \\
\hline OPQ 3.5 star $\times$ price & -0.2149 & 0.1382 & $-0.957 * * *$ & 0.1266 \\
\hline OPQ 4 star $\times$ price & -0.0751 & 0.1316 & $-0.4528 * * *$ & 0.1171 \\
\hline BID 3 star $\times$ price & 0.2818 & 0.1539 & $-2.3712^{* * *}$ & 0.3031 \\
\hline BID 3.5 star $\times$ price & 0.1233 & 0.1205 & $-0.9668 * * *$ & 0.2407 \\
\hline BID 4 star $\times$ price & 0.1296 & 0.1148 & $-0.7965 * * *$ & 0.2091 \\
\hline REG guest rating & 0.0845 & 0.0795 & 0.0798 & 0.0863 \\
\hline OPQ guest rating if shown & $0.1672 *$ & 0.0738 & $0.3625^{* * * *}$ & 0.1086 \\
\hline OPQ (shown or no shown) & -0.5448 & 0.3223 & -0.6267 & 0.3482 \\
\hline
\end{tabular}

Note. $\mathrm{MNL}=$ multinomial logit.

$*_{p}<.05$. $*_{p}<.01$. **** $<.001$.

Table 4.

Price Sensitivity for Each Channel.

\begin{tabular}{lccc}
\hline & REG & OPQ & BID \\
\hline Traditional & 0.0049 & 0.0086 & 0.0144 \\
Menu & 0.0054 & 0.0051 & 0.0286 \\
\hline
\end{tabular}

all negative which shows that lower priced hotel rooms are always more attractive to consumers no matter where they are listed. Moreover, we can compute the price sensitivity for each channel which is the utility change induced by one dollar value of the price change, and the results are summarized in Table 4 (note that we need to convert the standardized prices back to normal values). It appears consumers are more price sensitive toward opaque hotel listings than regular ones, that is quite intuitive and consistent with the empirical results in Granados et al. (2012).

By looking at coefficient estimates of hotel star variables, we can see that for REG listings, consumers prefer lower stars to higher, whereas for both OPQ and BID, hotels with higher stars are more popular than the ones with lower star ratings. These opaque findings are consistent with Anderson and Xie (2012) who indicated 4 star hotels have the largest transaction share on Hotwire. Together these channel-star estimates imply that opaque consumers prefer the value trade-off of less expensive higher star hotels, whereas as full information consumers can not afford or choose not to purchase higher priced higher star classified hotels.

To test the impact of star level on price sensitivities across different channels, we included an interaction term, that is, the Star $\times$ Price variables in the MNL model. For example, for a 3 star hotel listed on REG, adding the coefficients of price and Star $\times$ Price variables together and taking the absolute value, we obtain the new marginal utility for attribute price which is 0.8327 . Similarly, we compute the new marginal utilities for price for other stars and channels, and list the results in Table 5. As one can see that for both REG and OPQ, lower stars are associated with higher 
Table 5.

The New Marginal Utility for Price Attributes Across Different Channels.

\begin{tabular}{lccc}
\hline Star Rating & REG & OPQ & BID \\
\hline 3 & 0.8327 & 0.9632 & 0.3982 \\
3.5 & 0.6615 & 0.8203 & 0.5567 \\
4 & 0.4034 & 0.6806 & 0.5503 \\
4.5 & NA & 0.6055 & 0.6799 \\
\hline
\end{tabular}

Table 6.

Marginal Utility With Increasing Guest Rating.

\begin{tabular}{lcr}
\hline \multirow{2}{*}{$\begin{array}{l}\text { Increasing } \\
\text { Guest Rating }\end{array}$} & \multicolumn{2}{c}{ Marginal Utility \% } \\
\cline { 2 - 3 } & REG & OPQ \\
\hline 3.5 to 4 & 14.29 & 206.24 \\
4 to 4.5 & 12.5 & 67.35 \\
4.5 to 4.8 & 6.67 & 24.15 \\
\hline
\end{tabular}

price sensitives, that is, when consumers see a lower star hotel listed on these two channels, they tend to put more weight of their choice on price variables. However, interestingly, BID does not follow the same pattern as the other two channels, and price sensitivities rather fluctuate across the star ratings, which might be due to the connection between price and the probability of offers being accepted. For BID, consumers might choose a lower star hotel with a higher price but with a higher chance of winning over a higher star hotel with a lower price but with a lower chance of winning. Consumers are not only trading off stars and prices but also considering the chance of winning the bid, that is, the chance of getting the hotel room.

Prior guest rating or review scores impact OPQ options in two forms as they may (coded as 1 ) or may not (coded as 0 ) be present and then, if present, have differing scores. The marginal utility impact of the rating is then a combination of the shown dummy and the actual rating, that is, if the guest rating on OPQ is 3.5 , then the value to the utility is $3.5 \times$ $0.1672+(-0.5448)=0.0404$. This value is bigger than zero, that is, the value of the guest rating being not shown. This tells us showing guest rating attracts consumers more than not showing it. Similarly, we can compute the value added to the utility for guest rating being 4 , which is 0.1242 , and so the marginal increment percentage to the utility when guest rating increases from 3.5 to 4 given other attributes remaining the same is $(0.1242-0.0404) / 0.0404=206.24 \%$. Table 6 provides the marginal utility in percentages for both REG and OPQ alternatives indicating stronger impacts of ratings for opaque listings as compared with full information. The opaque consumer finds more utility (vs. the full information consumer) from user reviews as they considerably reduce the uncertainty in the option in the absence of typical information available at regular hotel listing. Interestingly, the marginal utility decreases with increasing ratings, indicating that once the guest rating is high enough (e.g., $\geq 4$ ), increasing guest rating will not have as large an impact on consumers choice as compared to with lower scores.

\section{Differences Across Survey Formats}

Table 3 summarizes parameter estimates from both survey formats with Figure 3 displaying a line plot of the coefficients for ease of comparison. As the table and figure indicate for the most part parameter estimates are very similar (in scale and significance). What is clear from Table 3 is the increased price sensitivity when consumers are allowed more flexible choices during the experiment. Although numerous price-related parameter estimates are not significant in the traditional experimental format, they become so in the menu format. This sensitivity is best shown in Table 4 where we summarize the price sensitivity across the three channels indicating that while REG and OPQ have similar sensitivities, the BID option is twice as price sensitive (for menu as compared with the traditional choice format) The increased price sensitivity of the BID channel resulting from more flexible price (bid) options impacts not only the BID channel but also the other two channels with the OPQ channel and REG channel showing small reductions in sensitivity. In the following section, we estimate the incremental revenue from opaque selling and highlight the impacts of this increased price sensitivity under the BID option.

\section{Incremental Demand and Revenue From Opaque Booking Channels}

Assume a hotel is currently selling via a traditional full information OTA and is looking to also sell via opaque OTAs. The REG, OPQ, and BID listings are priced at $p_{1}, p_{2}$, and $p_{3}$, respectively. Let $M S_{h}$ be the potential market share across these three channels, as discussed above, and without loss of the generality, we assume the market is normalized to be one customer, designated $n$. We also note that the hotel only gets a fraction of the demand for the opaque listings presented by the choice probability from the MNL model (with the remaining going to other hotels listing via the opaque OTAs). This is because the opaque listings' identities are concealed until after the purchase, and so the listing on opaque channels could be any hotel with the same characteristics within that area. We further can assume that this fraction is approximately just the hotel's market share $d$ introduced previously. Therefore, given the attributes of the three hotel listings and fraction of the demand for the opaque listings $d$, total demand for hotel from selling through REG, OPQ, and BID is $D_{1}=P_{n 1}+d P_{n 2}+d P_{n 2}$ with total expected revenue: 


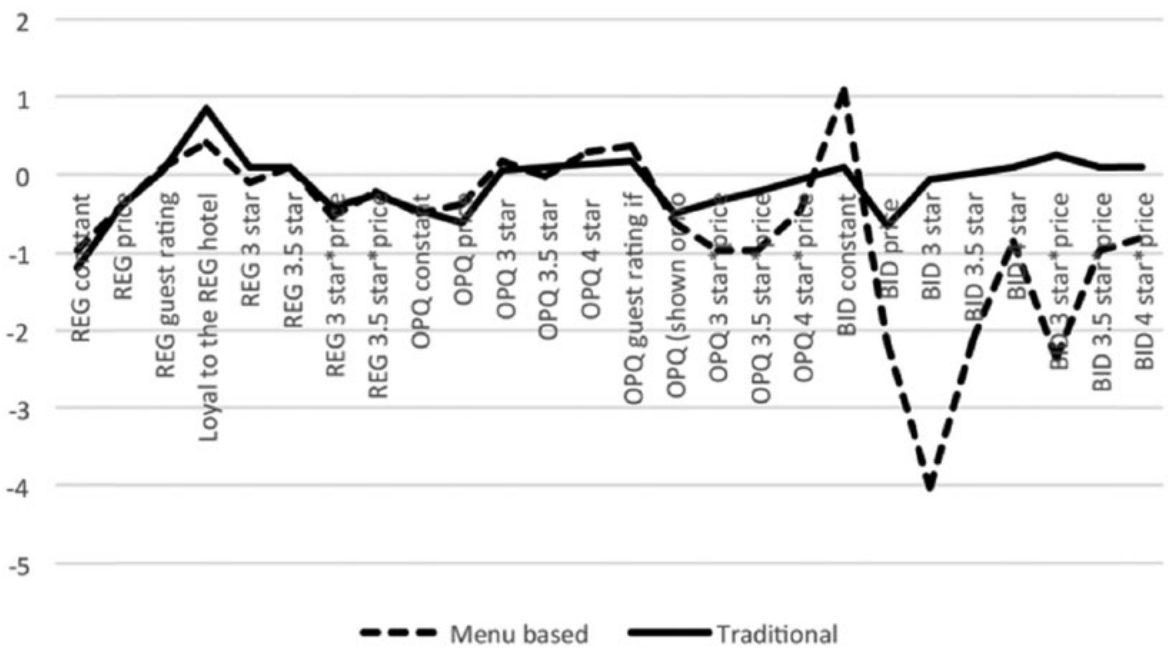

Figure 3.

Comparison of Traditional Versus Menu-Based Parameter Estimates.

$$
\pi_{1}=p_{1} P_{n 1}+\sum_{j=2}^{j=3} d p_{j} P_{n j},
$$

where $P_{n j} j=1,2,3$ are given in Equation 8. If the hotel decides to only list a room on the regular full information channel, then by the IIA property mentioned previously, the choice probability for the REG alternative needs to be proportionally adjusted so that the sum of it and the choice probability of the "No Purchase" alternative is still 1. Thus, we have

$$
P_{n 1}^{\prime}=\frac{e^{\beta_{1}^{\prime} \mathbf{X}_{n 1}}}{e^{\beta_{1}^{\prime} \mathbf{X}_{n 1}}+e^{U_{00}}},
$$

where $U_{00}$ is given in Equation 7. So the corresponding total expected revenue that the hotel can now obtain is

$$
\pi_{2}=p_{1} P_{n 1}^{\prime},
$$

where $P_{n 1}^{\prime}$ is given by Equation 10 .

We now compute the hotel's demand and revenue from selling through offline channels. Using the earlier discussed STR Global channel share estimates, the offline channel market share equals 1 minus online market shares or $75.9 \%$. Hence, our hotel's actual offline demand is $75.9 \%$ multiplied by our hotel's market share $d$. Similar to the method of computing no-purchase utility, we use this actual offline demand to estimate the offline demand in the model. Recall the our hotel's actual REG demand $=(17.5 \%+4.6 \%) d$, and its estimate in the model is the choice probability of REG hotel listing, that is, $P_{n 1}$ given in Equation 8, so the offline demand in the model is $D_{\text {off }}=$ actual offline demand $\times$ REG choice probability / actual REG demand $=75.9 \% \times P_{n 1} /$ $22.1 \%$. This implies that adding opaque listing will also dilute the offline channel demand. As usually the price of offline channels is the same as online channels (REG), so the offline revenue is denoted by $\pi_{o f f}=D_{o f f} \times p_{1}$. Therefore, adding opaque listings (OPQ and BID) to a REG listing gives a potential incremental demand of $\left(P_{n 1}+d P_{n 2}+d P_{n 2}\right.$ $\left.-P_{n 1}^{\prime}\right) /\left(P_{n 1}^{\prime}+D_{\text {off }}\right)$ and incremental revenue of $\left(\pi_{1}-\pi_{2}\right) /$ $\left(\pi_{2}+\pi_{o f f}\right)$. We use the following example to illustrate these calculations.

Consider a 4 star Hilton hotel selling rooms online through at traditional full information OTA. The hotel has a posted price of $\$ 349$ (USD) and a guest review score of 4.0. The hotel is considering listing a posted price opaque channel at a price of 175 per room/night as well as accepting bids at an opaque OTA with bidding at a level of $\$ 140$ per room/ night. The hotel's current market share averages $10 \%(d=$ 0.1 ). Using the equations above, the potential incremental demand from opaque selling is $5.87 \%$ and the corresponding incremental revenue is $2.23 \%$. Incremental revenue is less than incremental demand as some original full information consumers switch to opaque channels resulting in price dilution but this dilution is more than offset by addition volume. Using typical hotel performance terminology, the hotel receives an increase in occupancy through a decrease in average selling price or average daily rate which combine together to create an increase in revenue per available room (if prices are set properly on opaque channels).

Table 7 displays the average incremental demand and revenue from adding both OPQ and BID listings to a REG listing as estimated using parameter estimates from the traditional choice experimental format. The averages are taken across all possible alternatives' attributes (except for price) combinations for each BID and OPQ price pair ranging from $30 \%$ to $90 \%$ of the REG price. 
Table 7.

Average Incremental Demand and Revenue (Shaded) From Adding Both OPQ and BID Listings to a REG Listing (\%) via Traditional Choice Experiment.

\begin{tabular}{|c|c|c|c|c|c|c|c|c|c|c|c|c|c|c|}
\hline \multirow{3}{*}{$\frac{\text { Traditional }}{\text { BID/REG }}$} & \multicolumn{14}{|c|}{ OPQ Price/REG Price } \\
\hline & \multicolumn{2}{|c|}{0.3} & \multicolumn{2}{|c|}{0.4} & \multicolumn{2}{|c|}{0.5} & \multicolumn{2}{|c|}{0.6} & \multicolumn{2}{|c|}{0.7} & \multicolumn{2}{|c|}{0.8} & \multicolumn{2}{|c|}{0.9} \\
\hline & 4.65 & 1.24 & 4.47 & 1.42 & 4.3 & 1.51 & 4.15 & 1.53 & 4.02 & 1.49 & 3.92 & 1.43 & 3.83 & 1.35 \\
\hline 0.4 & 4.52 & 1.33 & 4.3 & 1.55 & 4.07 & 1.68 & 3.87 & 1.73 & 3.69 & 1.72 & 3.53 & 1.66 & 3.41 & 1.58 \\
\hline 0.5 & 4.42 & 1.35 & 4.15 & 1.61 & 3.87 & 1.77 & 3.61 & 1.85 & 3.37 & 1.85 & 3.16 & 1.80 & 2.99 & 1.72 \\
\hline 0.6 & 4.34 & 1.33 & 4.03 & 1.60 & 3.71 & 1.79 & 3.40 & 1.88 & 3.10 & 1.90 & 2.84 & 1.86 & 2.62 & 1.77 \\
\hline 0.7 & 4.28 & 1.29 & 3.94 & 1.57 & 3.58 & 1.76 & 3.23 & 1.86 & 2.89 & 1.89 & 2.58 & 1.85 & 2.31 & 1.77 \\
\hline 0.8 & 4.23 & 1.25 & 3.88 & 1.53 & 3.49 & 1.72 & 3.1 & 1.82 & 2.72 & 1.85 & 2.37 & 1.81 & 2.07 & 1.72 \\
\hline 0.9 & 4.2 & 1.2 & 3.83 & 1.48 & 3.42 & 1.67 & 3.01 & 1.77 & 2.6 & 1.79 & 2.22 & 1.74 & 1.89 & 1.65 \\
\hline
\end{tabular}

Table 8.

Average Incremental Demand and Revenue (Shaded) From Adding Both OPQ and BID Listings to a REG Listing (\%) via Menu base Choice Experiment.

\begin{tabular}{|c|c|c|c|c|c|c|c|c|c|c|c|c|c|c|}
\hline \multirow{3}{*}{$\begin{array}{l}\text { Menu } \\
\text { BID/REG } \\
0.3\end{array}$} & \multicolumn{14}{|c|}{ OPQ Price/REG Price } \\
\hline & \multicolumn{2}{|c|}{0.3} & \multicolumn{2}{|c|}{0.4} & \multicolumn{2}{|c|}{0.5} & \multicolumn{2}{|c|}{0.6} & \multicolumn{2}{|c|}{0.7} & \multicolumn{2}{|c|}{0.8} & \multicolumn{2}{|c|}{0.9} \\
\hline & 5.5 & 1.62 & 5.47 & 1.75 & 5.45 & 1.81 & 5.43 & 1.82 & 5.42 & 1.8 & 5.41 & 1.76 & 5.4 & 1.73 \\
\hline 0.4 & 5.29 & 1.7 & 5.18 & 2 & 5.06 & 2.19 & 4.96 & 2.28 & 4.86 & 2.29 & 4.78 & 2.24 & 4.72 & 2.17 \\
\hline 0.5 & 5.15 & 1.58 & 4.94 & 1.96 & 4.7 & 2.24 & 4.42 & 2.41 & 4.14 & 2.47 & 3.87 & 2.42 & 3.62 & 2.3 \\
\hline 0.6 & 5.09 & 1.49 & 4.84 & 1.88 & 4.52 & 2.17 & 4.14 & 2.36 & 3.7 & 2.43 & 3.25 & 2.38 & 2.8 & 2.22 \\
\hline 0.7 & 5.08 & 1.45 & 4.81 & 1.83 & 4.47 & 2.13 & 4.04 & 2.31 & 3.55 & 2.37 & 3.02 & 2.31 & 2.48 & 2.14 \\
\hline 0.8 & 5.07 & 1.43 & 4.8 & 1.82 & 4.45 & 2.11 & 4.02 & 2.29 & 3.51 & 2.34 & 2.95 & 2.27 & 2.39 & 2.09 \\
\hline 0.9 & 5.07 & 1.43 & 4.8 & 1.81 & 4.45 & 2.1 & 4.01 & 2.28 & 3.5 & 2.33 & 2.93 & 2.26 & 2.36 & 2.08 \\
\hline
\end{tabular}

As displayed in the table, incremental demand and revenue from adding opaque listings are positive across all discount levels. The positive incremental values indicate that adding opaque listings to the existing regular listing enables a natural price discrimination and increased market segmentation by reaching customers with different price sensitivities or valuation of the products. However, appropriate prices need to be set on these three channels to balance the revenue gain from having extra opaque demand and the revenue loss from diluting the revenue that we could have received from selling a full priced product through the regular channel. As seen in Table 7 that as prices of opaque listings decrease, incremental demand is increasing as more customers are able to purchase (i.e., there is an option below their willingness to pay). However, incremental revenue is not strictly increasing as opaque prices decrease, but first increasing, and then decreasing as after a while incremental demand is insufficient to offset dilution.

Table 7 indicates that incremental revenue is highest at $1.9 \%$ when the OPQ price is $70 \%$ and the BID price is $60 \%$ of the REG price. Table 8 shows similar incremental demand and revenue results obtained using parameter estimates from the menu-based choice experiment. The menubased model indicates the optimal posted opaque price remains at $70 \%$ of regular posted prices, but owing to increased price sensitivity, the optimal opaque bidding prices reduces to $50 \%$ with a resulting increase of $2.47 \%$ in total revenue. It is interesting to note that current industry practice shows hotels using slightly lower prices with posted opaque prices around $50 \%$ to $60 \%$ and accepted opaque bids around $40 \%$ to $50 \%$ of regular prices. These lower prices in practice are probably a result of hotels competing against each other for the opaque business versus setting optimal opaque prices (vs. regular full information prices).

\section{Summary and Managerial Insights}

Selling through opaque posted channels (e.g., Hotwire. com) or/and opaque bidding channels (e.g., Priceline.com) along with regular full information channels (e.g., Expedia. com) provides the service provider an opportunity to segment consumers, expand the market, and increase total revenue. On one hand, consumers who are less sensitive to prices and care to know the identity and location of the hotel will choose to book the room through the regular full information channel. On the other hand, price sensitive consumers who are not completely concerned about which hotel they stay exactly will yield to the option of trading the full information of the hotel for lower prices and book through 
opaque channels. Therefore, adding opaque channels to an existing regular full information channel enables the service provider to capture more consumers without heavily diluting the revenue (i.e., simply lowering prices at Expedia). Although this advantage of implementing opaque selling has received considerable attention in the literature, this article is the first that is dedicated to evaluate consumer preferences among these three channels and show the potential incremental revenue increment from selling through multiple types of opaque channels. We implement an online choice experiment with choice scenarios showing the hotel listings on the three channels with varying degrees of product opacity and analyze the experimental data. We use an MNL choice model to study consumers' preferences among channels and quantify the trade-offs consumers make during their hotel purchase decision.

We make contributions to the opaque selling literature and hotels in general across numerous fronts. From a hotel demand standpoint, as expected, purchase probabilities increase with decreasing prices but we illustrate that price sensitivity increases with increasing channel opacity. Similarly, while controlling for price, lower star classified hotels (vs. higher classified hotels) are more attractive to consumers on full information channels, the opposite is true for opaque channels with consumers preferring higher star hotels. Last, while guest reviews have a positive impact upon purchase decisions, this impact is amplified for opaque versus full information listings. Together these three findings illustrate that consumers are actively engaging in value seeking on opaque channels, they are very price sensitive and seek higher star hotels with better review scores in an effort to offset some of the uncertainty in not knowing the hotel they are purchasing until after the room is fully prepaid in advance. In addition to quantifying customer preferences across purchase channels, our MNL framework allows us to evaluate incremental revenue from opaque selling. Our incremental demand and revenue estimates provide reasonable and generalizable insights as we calibrate our choice model no-purchase probability using representative channel shares for domestic U.S. hotels. We find that both demand and revenue gains are achieved by adding opaque listings to the regular listing as the service provider is able to reach more customers with heterogeneous price sensitivities. However, the service provider needs to set appropriate prices across opaque and full information channels as deeply discounted opaque prices result in elevated levels of dilution but measurable discounts are required for consumers to overcome the uncertainty in opaque channels. We find that optimal incremental revenues are achieved with opaque posted prices at $70 \%$ and opaque bidding prices at about $60 \%$ of regular full information prices, slightly higher that prices we see in practice indicating that firms while growing demand with deeply discounted opaque prices may doing so at the cost of incremental revenues.
As discussed earlier, our article makes several contributions to the literature as we empirically assess the simultaneous use of posted opaque selling, opaque bidding in concert with transparent or full information selling. We perform this comparison in a hotel setting which given its larger degree of product differentiation (vs. airlines) runs more risk of demand dilution through opaque selling. We show that even in the face of potential dilution, there exists as set of prices across opaque (bidding thresholds for opaque bidding) and transparent channels that maximize firm revenue. Our insights are for firms without capacity constraints, and we would expect opaque prices (and thresholds) to approach transparent prices as demand approached capacity. Last, we illustrate some of the pitfalls of traditional choice experiments as we show slight changes in product attributes and channel choice under the more flexible structure of menu-based choice analysis.

\section{Declaration of Conflicting Interests}

The author(s) declared no potential conflicts of interest with respect to the research, authorship, or publication of this article.

\section{Funding}

The author(s) received the following financial support for the research, authorship, or publication of this article: Paper is supported by National Natural Science Foundation of China grant 71302079 .

\section{References}

Anderson, C. K. (2009). Setting prices on priceline. Interfaces, 39, 307-315.

Anderson, C. K., \& Xie, X. (2012). A choice-based dynamic programming approach for setting opaque prices. Production and Operations Management, 21, 590-605.

Anderson, C. K., \& Xie, X. (2014). Pricing and market segmentation using opaque selling mechanisms. European Journal of Operational Research, 233(1), 263-272.

Ben-Akiva, M., \& Lerman, S. R. (1985). Discrete choice analysis: Theory and application to travel demand. Cambridge, MA: The MIT Press.

Fay, S. (2008). Selling an opaque product through an intermediary: The case of disguising one's product. Journal of Retailing, 84, 59-75.

Granados, N., Gupta, A., \& Kauffman, R. (2008). Designing online selling mechanisms: Transparency levels and prices. Decision Support Systems, 45, 729-745.

Granados, N., Gupta, A., \& Kauffman, R. (2012). Online and offline demand and price elasticities: Evidence from the air travel industry. Information Systems Research, 23, 164-181.

Hensher, A. D., Rose, J. M., \& Greene, W. H. (2005). Applied Choice Analysis. Cambridge, England: Cambridge University Press.

Kamakura, W. A., \& Kwak, K. (2010). Menu-choice modeling (Working paper). Durham, NC: Fuqua School of Business, Duke University.

Liechty, J., Ramaswamy, V., \& Cohen, S. H. (2001). Choice menus for mass customization: An experimental approach 
for analyzing customer demand with an application to a webbased information service. Journal of Marketing Research, 36, 183-196.

Louviere, J. J. (1992). Experimental choice analysis: Introduction and overview. Journal of Business Research, 24, 89-95.

Ratliff, R. M., Rao, B. V., Narayan, C. P., \& Yellepeddi, K. (2008). A multi-flight recapture heuristic for estimating unconstrained demand from airline bookings. Journal of Revenue and Pricing Management 7, 153-171.

Shapiro, D., \& Shi, X. (2008). Market segmentation: The role of opaque travel agencies. Journal of Economics \& Management Strategy, 17, 803-837.

Shapiro, D., \& Zillante, A. (2009). Naming your own price mechanisms: Revenue gain or drain? Journal of Economic Behavior \& Organization, 72, 725-737.

\section{Author Biographies}

Xiaoqing (Kristine) Xie is an Associate Professor of Operations Management at China Europe International Business School
(CEIBS). She received her $\mathrm{PhD}$ in operations management from the School of Hotel Administration, Cornell University. Her papers have been extensively published in leading academic journals including Production and Operations Management, European Journal of Operational Research, Decision Sciences, Cornell Hospitality Quarterly, and International Journal of Revenue Management.

Chris K. Anderson is an Associate Professor at Cornell University's School of Hotel Administration and Director of Cornell's Center for Hosptilaity Reseach. He teaches and conducts research in data analytics, pricing, distribution and revenue management.

Rohit Verma has been a faculty member at Cornell University's School of Hotel Administration since 2006. He is currently the Dean of External Relations for Cornell College of Business, Executive Director of the Cornell Institute for Healthy Futures, and Singapore Tourism Board Distinguished Professor in Asian Hospitality Management. 